\title{
Adverse psychiatric effects of non-psychotropic medications
}

\author{
Ankit Gupta \& Rakesh K. Chadda
}

\begin{abstract}
SUMMARY
Patients presenting to psychiatrists frequently have comorbid medical conditions for which they are receiving treatment. A range of medications used for treatment of these conditions can have adverse effects resembling psychiatric symptoms. This article presents the results of our review of the literature on psychiatric adverse effects of various non-psychotropic medications, and discusses the mechanisms of such effects, their assessment and management. Among the commonly prescribed drugs found to have psychiatric adverse effects are corticosteroids, anti-Parkinsonian drugs, antiepileptics, antiretrovirals, antibiotics, anticancer drugs, analgesics, drugs targeting endocrine and cardiovascular disorders, immunosuppressants, skeletal muscle relaxants and bronchodilators. Some adverse effects are predictable and dose dependent, whereas others are rare and idiosyncratic, and psychiatrists need to be aware of them for accurate diagnosis and appropriate treatment.
\end{abstract}

\section{LEARNING OBJECTIVES}

- Appreciate the existing evidence and methodological issues in studying adverse psychiatric effects of non-psychotropic drugs

- Improve knowledge about adverse psychiatric effects of commonly prescribed drugs for various medical disorders

- Develop a structured approach towards diagnosing and managing adverse psychiatric effects of non-psychotropic drugs

\section{DECLARATION OF INTEREST}

None

Adverse psychiatric effects are unintended and potentially harmful mental and behavioural symptoms and syndromes resulting from administration of a drug for therapeutic purposes. Such adverse effects have been well studied and recognised in the context of psychotropic medications and psychoactive substances. However, many non-psychotropic medications regularly prescribed for various medical conditions are known to cause similar adverse psychological effects. Almost two-thirds of all the drugs included in the Physicians' Desk Reference (www.pdr.net) have potential psychiatric adverse effects. Further, with the advent of newer drug molecules and improved surveillance, the list of implicated medications is steadily increasing (Turjanski 2005; Rudorfer 2012). Mechanisms for the psychiatric effects of some drugs are well understood, but for others they are unexpected and only partially comprehended (Desai 2004; Turjanski 2005). Adverse effects can occur at usual therapeutic doses as well as at toxic doses, and they may resemble symptoms seen in other psychiatric disorders (Tango 2003). These psychiatric symptoms are wide ranging and include non-specific behavioural changes, anxiety, depression, frank psychotic symptoms, compulsive gambling and suicide (Holvey 2010). The symptoms usually remain unrecognised in medical settings and become apparent only when they are severe or interfere with treatment. In clinical practice, recognition, diagnosis and management of psychiatric symptoms in patients with medical conditions poses a challenge for both physicians and psychiatrists. Early identification of these adverse effects and prompt intervention may significantly reduce the healthcare burden and associated psychological distress.

\section{Harmful consequences of adverse psychiatric effects}

The unwanted psychiatric effects of commonly prescribed non-psychotropic drugs may have harmful consequences in the form of increased emotional suffering and subjective distress on the part of the patient (Desai 2004; Parker 2012). Further consequences may include increased length of hospital admission and increased utilisation of healthcare services and resources. Occurrence of severe psychiatric adverse effects during medical treatment can also increase the risk of disability, morbidity and even mortality (Desai 2004). Major symptoms such as agitation, psychosis and depression also carry a significant risk of harm or aggression towards self and others. Such aggressive or suicidal behaviours may sometimes warrant an urgent psychiatric admission, as they are unlikely to be managed
Ankit Gupta is a Senior Resident in the Department of Psychiatry at the All India Institute of Medical Sciences, New Delhi. Rakesh K. Chadda is Professor of Psychiatry at the All India Institute of Medical Sciences, New Delhi. His area of interest includes consultationliaison psychiatry, somatisation, and community and forensic psychiatry Correspondence Professor Rakesh K. Chadda, Department of Psychiatry, All India Institute of Medical Sciences, New Delhi 110029, India. E-mail: drrakeshchadda@gmail.com 
easily in non-psychiatric in-patient or out-patient settings. Intolerable or unmanageable adverse effects may further lead to discontinuation of the primary therapy and complicate recovery from the underlying medical condition (Turjanski 2005). Untreated psychiatric symptoms are also implicated in poor adherence to medical treatment in the long term (Pachi 2013).

\section{Problems of identifying adverse psychiatric effects}

Adverse psychiatric effects are often poorly characterised in clinical drug trials, because they are usually rare events in standard clinical practice (Holvey 2010). The majority of these effects only become apparent after licensing of drugs and widespread clinical use, and in postmarketing surveillance (Holvey 2010).

There are several methodological challenges in studying psychiatric adverse effects during drug development. The absence of commonly accepted and available animal models of mental disorders and symptomatology makes it difficult to study these effects in preclinical phases (Rudorfer 2012). Pre-marketing clinical trials are also of a shortterm nature and thus do not offer an opportunity to observe adverse effects that emerge after months or even years (Rudorfer 2012). In the recent past, reports of increased risk of suicidal thoughts and behaviour during treatment with some newly developed drugs have provoked concern among consumers regarding drug safety (Meyer 2010). These findings brought a much-needed focus on the necessity of considering potential psychiatric complications of new drugs at an earlier stage of development (Meyer 2010). Regulatory agencies have mainly responded by issuing appropriate warnings for prescribing physicians and consumers (US Food and Drug Administration 2009). Several new drugs have either been withdrawn by manufacturers (e.g. the anti-obesity drug rimonabant and the antitubercular drug iproniazid) or received warnings from regulatory agencies in recent years (e.g. the anti-smoking drug varenicline, anti-acne drug isotretinoin and anti-epileptic drugs) owing to their adverse psychiatric effects (Rudorfer 2012). However, intangible evidence of such a risk for many drugs (e.g. anti-epileptics) is still missing, owing to a lack of formal prospective post-marketing testing or trials for psychiatric adverse effects.

\section{Our literature search}

We conducted a literature search for psychiatric adverse effects of non-psychotropic medication using the Medline (PubMed), PsycINFO and
Scopus databases from inception until June 2015. Owing to the non-specificity of the term 'nonpsychotropic medication', several other terms, including 'nonpsychotropics', 'non-psychiatric drugs', 'commonly prescribed drugs' and the names of specific drug classes (e.g. corticosteroids, antiretrovirals), were used to extract previous reviews and original papers. Other terms used for searching in PubMed included 'psychiatric', 'neuropsychiatric', 'adverse events', 'side effects', 'complications' and specific syndromes such as 'mania', 'depression' and 'psychosis'. Published textbooks on consultation-liaison psychiatry were also searched for information on psychiatric adverse effects. To maintain clinical relevance, the focus of this review was limited to either the most commonly used drugs or those commonly associated with psychiatric adverse effects.

\section{Mechanism of adverse effects}

Drugs belonging to different classes act on the central nervous system (CNS) through various pathways, leading to a spectrum of neuropsychiatric adverse effects. The pharmacological mechanisms implicated in neurotoxicity or neuropsychiatric adverse effects of drugs can be broadly categorised into pharmacodynamic and pharmacokinetic mechanisms. Pharmacodynamic mechanisms involve the modification of major neurotransmitter systems by the drug molecule. The mode of action may involve a direct influence on neurotransmitter systems (e.g. dopamine agonists, interleukins) or it may occur in a more indirect manner, such as corticosteroids or sex steroids acting on the hypothalamus-pituitary-adrenal axis (Tango 2003). Pharmacokinetic mechanisms are relevant in the case of drugs that follow a known doseresponse curve. Low clearance of the drug owing to disease state, hepatic enzyme polymorphisms and drug interactions leading to metabolic inhibition are the main pharmacokinetic mechanisms (Ferrando 2010). The risk of neuropsychiatric effects, as well as other adverse effects, may increase as the concentration of the drug (e.g. mefloquine, macrolides) increases.

Risk factors for development of psychiatric adverse effects (listed in Box 1) may be related to the treatment regimen or certain specific patient characteristics (Alomar 2014). Drugs with a narrow therapeutic index have a higher propensity to cause adverse effects. Complex treatment regimens involving multiple medications from different classes increase this risk owing to possible synergistic actions and pharmacokinetic interactions. Polypharmacy is common, especially in the case of older patients, and may involve 
B0X 1 Risk factors for development of psychiatric side-effects

\section{Treatment-related factors}

- Polypharmacy and complex treatment regimens

- High dosage of drugs

- Route of administration (e.g. intravenous, intrathecal)

- Rapid administration (by any route)

- Narrow therapeutic index of the drug

Patient-related factors

- Present or past mental illness

- Poor metabolic status

- Increased permeability of blood-brain barrier (e.g. meningitis)

- Very young or elderly patients

- Postpartum state

- Other stressful situations (e.g. intensive care unit)

several non-essential medications, increasing the risk of side-effects in general and psychiatric sideeffects in particular (Desai 2004). Intravenous or intrathecal route of administration, higher doses (e.g. of corticosteroids or antimicrobials) and a faster rate of administration also increase the risk of psychiatric adverse effects.

Among the patient characteristics, current mental illness or history of mental illness are important risk factors for higher incidence of psychiatric side-effects during treatment with the drug in question (Tango 2003). Specific patient populations, such as very young or elderly individuals and postpartum women, are also at increased risk (Alomar 2014). Furthermore, increased permeability of the blood-brain barrier and poor metabolic status of the patient (e.g. hepatic or renal insufficiency, slow metabolism or other metabolic disturbances) may also lead to higher drug concentrations and consequent adverse effects (Ferrando 2010). Knowledge of these risk factors is important in order for the clinician to suspect, identify and manage common or predictable psychiatric symptoms arising during medical treatment.

\section{Assessment}

The first step in the assessment of psychiatric adverse effects is establishing the link of causality between the medications and the adverse effects. However, several confounding factors may lead to difficulty in establishing causality: the adverse effects are rarely specific to the implicated drug, diagnostic tests are usually absent, and rechallenge with the same drug usually cannot be justified for ethical reasons. The World Health Organization (WHO) and the Uppsala Monitoring Centre (UMC) have developed a standardised causality assessment system for drug monitoring in consultation with the national centres participating in the programme on international drug monitoring (WHO 2005). The WHO-UMC system for standardised case causality assessment is meant as a practical tool for the assessment of case reports of medication adverse effects. Several factors (listed in Box 2) are considered important in ascertaining the strength of the causal relationship between the drug and the adverse effect (Edwards 2000). The first presentation of psychiatric symptoms in a patient with pre-existing medical illness requires detailed assessment and investigation. Assessment should consist of focused psychiatric and medical history-taking, examination and investigations. All biological and psychosocial risk factors for the development of psychiatric symptoms in a given individual should be identified and considered. Particular attention should be paid to making a detailed chronological treatment review in order to identify the 'certain' or 'probable' offending drug.

\section{Differential diagnosis}

Psychiatrists have to be careful in differentiating various possible causes of presenting symptoms. Differential diagnoses should include probable physical illnesses with psychiatric symptoms (e.g. multiple sclerosis, metastasis, electrolytic disturbances), aggravation of pre-existing psychiatricillness, first psychiatric decompensation in individuals with no evident susceptibility, as well as adverse psychiatric side-effects at usual doses and intoxication-related or withdrawalrelated adverse effects (e.g. after discontinuation of anti-Parkinsonian agents, benzodiazepines, antidepressants, anabolic androgen steroids) (Tango 2003).

\section{BOX 2 Factors determining causal relationships between medications and psychiatric side-effects}

- Temporal relationship between the drug exposure and the psychiatric side-effect

- Definitive pharmacological or phenomenological evidence of specific psychiatric side-effects

- Presence or absence of alternative explanations for symptoms (e.g. disease, other drugs)

- Response to withdrawal of drug

- Effect of rechallenge with the same drug 


\section{Important drugs with adverse psychiatric effects}

Table 1 lists psychiatric adverse effects of major non-psychotropic drug groups prescribed in clinical practice.

\section{Chemotherapies}

Cancer patients frequently suffer mental and behavioural changes, which can present at any stage of the illness. The changes may be multifactorial in origin, i.e. secondary to the disease process, adverse effects of the treatment and the psychological distress associated with cancer diagnosis (Holland 2013). It is thus difficult to recognise the adverse effects and specifically attribute them to a drug from the prescribed combination of chemotherapy.

Almost all chemotherapeutic agents are associated with significant psychiatric adverse effects. Commonly reported adverse effects include cognitive impairment $(\approx 75 \%)$, delirium or confusional state $(\approx 30 \%)$ and psychotic symptoms (Ferrando 2010; Janelsins 2011). Predisposing factors such as age, dementia, functional impairment, nature and severity of illness, and malnutrition increase the risk of experiencing delirium during hospital admissions (Holland 2013). Mood symptoms or disorders (manic or depressive), anxiety symptoms, agitation and suicidal behaviour may also be seen but are less common (Ferrando 2010; Holland 2013). Some chemotherapeutic agents, such as vincristine, vinblastine, procarbazine, asparaginase and tamoxifen, may produce depressive symptoms (Mehta 2015). Delirium has been associated with methotrexate (intrathecal or intravenous), 5-fluorouracil, vincristine, vinblastine, bleomycin, carmustine, cisplatin, asparaginase, procarbazine, cytosine arabinoside and ifosfamide (Mehta 2015).

'Chemobrain' or cancer therapy-associated cognitive change is a phenomenon identified in patients who have received chemotherapy and hormonal therapy and have experienced difficulty in executive functions, multitasking, short-term memory recall and attention (Mehta 2015). These cognitive changes seem to be dosedependent, and certain chemotherapeutic agents, including methotrexate, fludarabine, cytarabine, 5-fluorouracil and cisplatin, are associated with worse cognitive effects (Wefel 2015).

If the psychiatric symptoms are severe, active psychiatric intervention is required. Psychotropic drugs must be carefully selected to avoid adverse interactions with chemotherapeutic agents, including interactions that potentially limit the therapeutic efficacy of chemotherapy (Holland 2013).

\section{TABLE 1 Important psychiatric adverse effects of major drug groups prescribed in clinical practice}

\begin{tabular}{|c|c|c|}
\hline Drug group & Major implicated drugs & Important adverse effects \\
\hline Anticancer/chemotherapy & $\begin{array}{l}\text { 5-fluorouracil, asparaginase, bortezomib, ifosfamide, } \\
\text { vincristine }\end{array}$ & $\begin{array}{l}\text { More common: cognitive impairment, delirium, psychosis } \\
\text { Less common: depression, anxiety, suicidal ideation }\end{array}$ \\
\hline Immunomodulators & $\begin{array}{l}\text { Ciclosporin, corticosteroids, }{ }^{a} \text { interferon- } \alpha \text {, interleukins, } \\
\text { isotretinoin, tacrolimus }\end{array}$ & $\begin{array}{l}\text { More common: anxiety, insomnia, depression } \\
\text { Less common: mania, psychosis, agitation, delirium }\end{array}$ \\
\hline Cardiovascular drugs & $\begin{array}{l}\text { ACE inhibitors, alpha and beta adrenergic blockers, } \\
\text { anti-arrhythmics, }{ }^{\text {b }} \text { statins }\end{array}$ & $\begin{array}{l}\text { More common: fatigue, sleep disorders } \\
\text { Less common: depression, anxiety }\end{array}$ \\
\hline Anticonvulsants & Carbamazepine, levetiracetam, phenytoin, topiramate & $\begin{array}{l}\text { More common: sedation, cognitive impairment, agitation } \\
\text { Less common: depression, suicidal ideation, delirium }\end{array}$ \\
\hline Anti-Parkinsonians & Anticholinergics, dopamine agonists, ${ }^{c}$ entacapone & Agitation, sleep disorders, psychosis, delirium \\
\hline Antiretrovirals & Efavirenz, ritonavir, zidovudine & $\begin{array}{l}\text { More common: anxiety, fatigue, sleep disorders, depression } \\
\text { Less common: euphoria, agitation, psychosis, delirium }\end{array}$ \\
\hline Antitubercular antibiotics & Cycloserine, isoniazid, rifampicin & Sleep disorders, depression, psychosis, delirium \\
\hline Other antimicrobials & Mefloquine, metronidazole, quinolones & Anxiety, insomnia, psychosis, delirium \\
\hline Oral hypoglycaemics & Glimepiride, metformin & Anxiety, depression, irritability, cognitive impairment \\
\hline Anabolic and androgenic steroids & Testosterone and its derivatives & Irritability, mania, psychosis, dependence \\
\hline Antihistaminics & Cimetidine, promethazine & Sedation, agitation, psychosis, delirium \\
\hline Analgesics & Aspirin, ibuprofen, indomethacin & Sleep disorders, fatigue, agitation, anxiety, mood changes \\
\hline Surgery and critical care & Anaesthetics, propofol, suxamethonium & Cognitive impairment, delirium \\
\hline Respiratory system drugs & Beta adrenergic agonists, decongestants & Agitation, insomnia, euphoria, delirium \\
\hline Muscle relaxants & Baclofen, dantrolene & Anxiety, agitation, mood changes, delirium \\
\hline
\end{tabular}

ACE, angiotensin-converting enzyme.

a. Corticosteroids can also cause dependence.

b. Anti-arrhythmics can also cause delirium

c. Dopamine agonists can also cause dopamine dysregulation syndrome. 


\section{Immunomodulators}

Immunomodulator drugs modulate (i.e. stimulate or suppress) the activity of immune system and are widely used in clinical practice. These drugs are generally prescribed for the treatment of cancers, lymphomas and autoimmune disorders, as well as post-organ transplantation and in the management of allergies. They include cytokines (interferons), interleukins, calcineurin inhibitors, corticosteroids, retinoic acid compounds and antihistamines. Adverse effects of immunomodulators include mood disturbance, insomnia, cognitive impairment and delirium (Heinrich 2009).

Calcineurin inhibitors (ciclosporin, tacrolimus) are reported to produce neurotoxic effects in $40-60 \%$ of organ transplant recipients (Bechstein 2000). The risk increases with biochemical and electrolyte derangements, intravenous drug administration or disruption of the blood-brain barrier (Bechstein 2000). Interferons and interleukins have been implicated in psychiatric adverse effects such as depression, anxiety, psychosis, suicidal ideation, hypomanic symptoms and cognitive impairment (Myint 2009). Interferons are frequently associated with affective disturbance, with depressive disorders seen in $16-58 \%$ of individuals. The disturbance may appear weeks or months into treatment and is more frequently associated with high-dose therapy (Raison 2005; Myint 2009). Depressive disorders are more of a concern with interferon- $\alpha$ (especially interferon$\alpha 2 b$ ) than interferon- $\beta$ (Raison 2005). Isotretinoin is known to be associated with depression, suicidal ideation and psychosis (Borovaya 2013). The frequency of depressive disorders during treatment with this drug varies from 1 to $11 \%$ in different studies, but it is unclear whether this is a consequence of isotretinoin therapy (Borovaya 2013). The effect is not known to be related to dose or duration of treatment, or to previous psychiatric illness. Antihistamines are known to be associated with sedation, cognitive impairment, agitation, psychosis and delirium (Turjanski 2005; Simons 2008). These effects are more pronounced with first-generation $\mathrm{H}_{1}$ antagonists and are rarely seen with newer agents (Abramowicz 2008; Simons 2008). $\mathrm{H}_{2}$ antagonists (especially cimetidine) have a risk of causing cognitive impairment and delirium in elderly and seriously ill patients (Abramowicz 2008).

Corticosteroids are the most commonly prescribed immunomodulators and are used for a variety of medical conditions. Psychiatric complications are not rare and range from clinically significant anxiety, insomnia, emotional lability, irritability and cognitive impairments to severe mood disorders, psychotic disorders, delirium and reversible dementia (Kenna 2011; Judd 2014). Dependence is an unusual but serious adverse effect of corticosteroid use and is based on the propensity of these drugs to induce euphoria as well as a characteristic withdrawal syndrome, in addition to directly influencing reward circuitry (Kenna 2011). Transient mild to moderate subsyndromal psychiatric symptoms are seen in approximately $13-62 \%$ of patients, while the incidence of major or severe symptoms ranges from 5 to $10 \%$ across studies (Warrington 2006). These adverse effects have a rapid onset after starting corticosteroids (usually within 1-2 weeks), and the likelihood of psychiatric symptoms follows a striking dose-response correlation. Hypomania and mania are the most common acute adverse effects, while the risk of depression increases after long-term or chronic corticosteroid therapy (Kenna 2011; Judd 2014). The risk of adverse effects is not significantly associated with history of psychiatric disorder or prior episodes of steroidinduced psychiatric symptoms (Kenna 2011).

\section{Cardiovascular drugs}

Numerous medications acting on the cardiovascular system have been associated with neuropsychiatric adverse effects, ranging from mood symptoms to cognitive effects to psychosis (Huffman 2007). Among the antihypertensives, calcium channel blockers, angiotensin-converting enzyme (ACE) inhibitors and angiotensin II receptor antagonists are associated with mood symptoms, psychosis and delirium, although the rates are low (Ferrando 2010; Parker 2012). However, diuretics may cause confusion and delirium secondary to electrolyte imbalance. Centrally acting agents such as clonidine, methyldopa and reserpine are consistently associated with fatigue and sedation (Huffman 2007). Depression was previously thought to be associated with beta-blockers (especially propranolol), methyldopa and reserpine. However, recent syntheses of data suggested that these associations are much weaker than originally believed (Celano 2011; Parker 2012). Fatigue and sedation are common adverse effects with clonidine (35\%), beta-blockers (>10\%) and ACE inhibitors (>5\%) (Sidhu 2008). Several anti-arrhythmic drugs are associated with psychosis and delirium. Lipidlowering drugs (statins) have previously been the focus of controversy, owing to concern regarding suicidal ideation and behaviour; however, several large prospective studies and meta-analyses failed to establish a causal association (Celano 2011). Most of the literature on major adverse effects 
of cardiovascular drugs is based on case reports and case series, and results from the few available well-controlled trials are mixed and inconclusive. Cardiovascular drugs may not consistently cause neuropsychiatric symptoms in the general population, although idiosyncratic reactions are possible (Huffman 2007).

\section{Anticonvulsants}

Adverse psychiatric effects associated with anticonvulsants are observed in about $15-20 \%$ of patients with epilepsy (Perucca 2012). Major effects of these drugs are behavioural and personality changes, mood disorders (especially depression), suicidal behaviour and psychosis (Ferrando 2010). In early 2008, the US Food and Drug Administration (FDA) issued a warning of an increased risk of suicidal ideation and behaviour during treatment with anticonvulsant drugs. The warning was based on a meta-analysis of 199 trials focusing on 11 anticonvulsants prescribed for various conditions, including epilepsy and psychiatric disorders, and later in the same year it was amended to include all anticonvulsant drugs (FDA 2008). However, no black box warning or prescription restrictions were imposed. Subsequent efforts at replicating the FDA metaanalysis and conclusions primarily by means of post-marketing observational studies have had mixed results. The risk of developing adverse psychiatric effects varies considerably among anticonvulsants, with comparatively higher risks associated with barbiturates, vigabatrin, tiagabine, topiramate, levetiracetam, zonisamide and felbamate (Perucca 2012). Recent evidence suggests that individual susceptibility plays an important part; for example, a positive psychiatric history is reported as an important risk factor for developing adverse psychiatric effects with some anticonvulsants (e.g. topiramate, levetiracetam) (Perucca 2012). The extent of suicide risk associated with anticonvulsants remains an open question, requiring caution and a high level of suspicion on the part of the clinician.

\section{Anti-Parkinsonian drugs}

Almost all anti-Parkinsonian drugs are associated with psychosis (20-30\%), sleep disturbance, cognitive impairment and mood changes (Zahodne 2008). Patients with advanced disease, prolonged duration of treatment, cognitive impairment and dyskinesias are at higher risk of developing these adverse effects (Ferrando 2010). Impulsive and compulsive behaviours, including pathological gambling, hypersexuality, compulsive shopping, compulsive eating, excessive engagement in hobbies, punding and dopamine dysregulation syndrome are other serious side-effects of dopaminergic medications (Raja 2012). The estimated prevalence of impulse control disorders is as high as $14 \%$ among patients with Parkinson's disease treated with these medications (Raja 2012). The propensity to develop adverse effects is highest with anticholinergics, followed by amantadine, dopamine agonists and catechol$O$-methyltransferase (COMT) inhibitors, and is least with the use of levodopa (Zahodne 2008). For management of adverse effects, drugs are sequentially reduced or withdrawn. When this strategy is not successful, antipsychotic treatment may be more appropriate. Quetiapine is the antipsychotic of choice in such cases, owing to more favourable tolerability than clozapine and other antipsychotics.

\section{Antiretroviral drugs}

Antiretroviral drugs are known to be associated with a wide range of symptoms, varying from sleep disturbance and mood disorders to cognitive impairment and psychosis (Abers 2014). Establishing aetiology is challenging because of the considerable overlap between the adverse effects of antiretroviral drugs and the complications of HIV infection. Variable cerebrospinal fluid penetration of individual antiretroviral drugs may contribute to differences in potential effects (the greatest effects are found with efavirenz, and the least with tenofovir) (Suvada 2013). Adverse effects are usually dose related. Patients with a psychiatric history are more vulnerable. Among the antiretrovirals, efavirenz has been associated with the highest risk of adverse effects (>50\%), with depression and sleep disorders being the most common effects (Abers 2014). These significant and distressing adverse effects may lead to poor adherence and interruption of antiretroviral therapy. Pharmacological management of these effects is complicated by significant interactions between antiretroviral and psychotropic drugs, which may necessitate withdrawal of a drug or change in treatment regimen.

\section{Antitubercular drugs and other antibiotics}

Adverse reactions have been mainly reported with cycloserine (9.7-50\%), isoniazid (1.9\%), ethionamide (1-2\%) and fluoroquinolones (14.4\%) (Pachi 2013). The risk of adverse reactions is increased with age, malnutrition, history of hepatitis, and HIV or hepatitis $\mathrm{C}$ virus infection (Pachi 2013). Several classes of antibiotics have side-effects ranging from minor confusion and irritability to severe encephalopathy and suicide 
(Turjanski 2005). Delirium is the most common adverse effect and is seen especially at high doses and in the presence of other risk factors. Quinolones and penicillins have higher risk of causing major psychiatric symptoms, including psychosis (Ferrando 2010). Amoxicillin (a penicillin-group antibiotic) is among the top ten most commonly prescribed drugs associated with psychiatric sideeffects (Hubbard 1991). Commonly prescribed antimalarial drugs (mefloquine, chloroquine etc.) have also been reported to be associated with psychiatric adverse effects, including sleep disorders, anxiety and depression (Holvey 2010).

\section{Drugs for endocrine disorders}

Apart from corticosteroids, relatively little information is available regarding the psychiatric adverse effects of drugs acting on the endocrine system. Adverse effects of oral hypoglycaemics are secondary to hypoglycaemia or their insulin-like psychiatric effects, which include anxiety, dysphoria, irritability and confusion (Ferrando 2010). Previous reports have suggested that hormone replacement therapy and oral contraception may be associated with negative mood changes in women, but findings have been inconsistent. However, more recent findings indicate the potential benefits of these therapies in the prevention of depression among women (Keyes 2013; Gordon 2014; Cheslack-Postava 2015). There is evidence in support of oestrogenbased therapies in the treatment of depression among perimenopausal and early postmenopausal women, but not women who are well into the postmenopausal period (Gordon 2014). Antithyroid medications have not been documented to cause any psychiatric side-effects. Good-quality evidence suggests that supraphysiological doses of anabolic steroids can directly cause hypomanic or manic symptoms, extending to aggression and violence (Kersey 2012). The risk of dependence and development of depressive symptoms on drug withdrawal has also been documented (Kersey 2012). Dopamine agonists used for hyperprolactinaemia also carry a risk of psychosis.

\section{Analgesics}

Non-steroidal anti-inflammatory drugs (NSAIDs) are frequently used in clinical practice, accounting for approximately $5-10 \%$ of all drug prescriptions (Onder 2004). Psychiatric symptoms are rare but relevant, considering the common use of these drugs. Major adverse effects include changes in cognition, mood state (rarely psychosis), sleep disturbance, and precipitation or exacerbation of pre-existing psychiatric disorders. The underlying mechanism is unclear, although it is hypothesised to be a probable consequence of impairment in neurotransmission modulated by prostaglandins in susceptible individuals (Onder 2004). Therefore, NSAIDs should be used with caution in high-risk individuals with pre-existing psychiatric illness and in the postpartum period. NSAID-related psychiatric adverse events have most commonly involved indomethacin and selective COX-2 inhibitors.

\section{Surgery and critical care}

Post-operative cognitive dysfunction is not uncommon after a major surgery and is usually short lived (Parker 2012). It is more common with general rather than regional anaesthesia, especially in the presence of other risk factors and medications. Malignant hyperthermia is a rare, potentially fatal adverse effect of some anaesthetics and neuromuscular blockers in genetically susceptible individuals (Ferrando 2010). Delirium is a major adverse effect of drugs commonly used in surgery and critical care.

\section{Respiratory drugs and muscle relaxants}

Beta agonists used for bronchial asthma and chronic obstructive pulmonary disease may lead to psychiatric symptoms secondary to their sympathomimetic actions (Sidhu 2008). Over-thecounter drugs used for respiratory conditions, such as combinations of antihistamines and decongestants, can potentially lead to psychosis and delirium (Abramowicz 2008). Skeletal muscle relaxants may also induce a variety of psychiatric symptoms.

\section{Herbal remedies}

Various alternative medicinal products are used by patients for a wide range of physical and psychiatric conditions, either alone or concomitant with allopathic medicine. With a few exceptions, there is a lack of systematic research and reliable data about the efficacy and safety of these herbal medicines (Ernst 2003, 2007; Bersani 2015). However, data are gradually accumulating data on adverse effects of some of these drugs, which are widely available without prescription over the counter and online. These effects may be associated with the primary ingredient or the contaminants found in the preparations. Confusion, encephalopathy, psychotic symptoms, drowsiness and mood disturbances have been reported in the literature, although their exact prevalence is unknown (Ernst 2003, Bersani 2015). Panax ginseng, ephedra, yohimbine, jimson weed, passionflower, liquorice, kava, ginkgo and St John's 
wort are some of the implicated herbal remedies (Ernst 2003; Bersani 2015). Use to the extent of dependence has been reported with products with stimulant-like properties (e.g. ephedra, yohimbine) (Bersani 2015).

\section{Principles of management}

Non-psychotropic medication-induced psychiatric symptoms can be encountered in diverse clinical situations. These symptoms are often a focus of attention for psychiatrists working in general hospital psychiatry, more commonly in consultationliaison settings such as medical wards, intensive care units (ICUs) and emergency services. Therefore, a good knowledge of psychiatric adverse effects of commonly prescribed non-psychotropics is essential for general as well as consultationliaison psychiatrists. Such knowledge needs to be integrated into routine clinical practice for accurate diagnosis and appropriate management of these symptoms.

The management of non-psychotropic adverse effects involves close coordination with other medical specialties to formulate a structured management plan (Box 3). The general principles of management focus mainly on reducing risk factors for psychiatric adverse effects and optimisation of pharmacotherapy of the primary medical illness. In the case of severely ill patients, measures

BOX 3 Principles of management of medication-induced psychiatric adverse effects

- Establish the probability of psychiatric symptoms being induced by medication

- Work in close coordination with the primary treatment team and effectively communicate the management plan

- Perform a comprehensive psychiatric and medical assessment (especially for risk factors)

- Monitor and reduce the modifiable risk factors for development or maintenance of adverse effects

- Optimise and rationalise pharmacotherapy for the primary medical illness

- Reassure the patient and manage minor or transient symptoms with non-pharmacological approaches

- Decide whether to discontinue or switch drugs only after weighing the potential risks and benefits

- Prescribe psychotropic agents judiciously and monitor regularly for any emerging adverse effects

- Psychoeducate the patient and family members regarding aetiology of psychiatric symptoms and the management plan such as maintaining hepatic and renal function, correction of electrolyte disturbances, control of superimposed infections, increasing sensory stimulation and reducing stress in ICUs (e.g. with regular family visits or pain control) may help in reducing psychiatric symptoms. Similarly, pharmacotherapy for primary medical illness can be modified by either changing timings or reducing doses of the drugs associated with adverse effects, and by stopping unnecessary or non-essential drugs to avoid interactions. Major changes such as discontinuation or switching of medications may be required in the case of severe or life-threatening symptoms. This should be done after taking into account the relative risks and benefits of such interventions. Generally, the 'offending' drug is switched to another drug if an equally effective but safer alternative is available. If no safer alternatives are available, and pharmacotherapy for primary illness is essential, then psychotropic drugs should be used for management. Switching to less effective alternatives may be considered in the case of persisting severe adverse effects, if psychotropic agents are either not tolerated or ineffective in controlling symptoms.

The psychiatric management plan should be communicated effectively and explicitly to the treating medical specialists. Psychotropic agents should be used cautiously for management in such cases, with gradual increments in doses and targeted minimum effective doses. In addition to the usual factors, the choice of psychotropic agent in such patients depends on its interaction with both the primary medical illness as well as the non-psychotropic drugs. Closer monitoring for adverse effects of psychotropic drugs is also important, owing to a higher risk in patients with severe medical illnesses. Psychoeducation aimed at the patient and family members is also required to help patients make accurate attributions and conclusions concerning their psychological changes. A comprehensive psychoeducation programme helps in avoiding the stigma, distress and other costs of unjustified long-term psychiatric treatment.

\section{Conclusions}

Numerous drugs used for non-psychiatric illnesses are linked with adverse psychological effects (Table 1). It is important for psychiatrists to recognise such effects and differentiate them from other causes. For the majority of these drugs, psychological adverse effects are relatively infrequent and usually do not interfere with treatment. However, some drugs, including steroids, dopamine agonists, interferons, efavirenz 
and isoniazid, frequently lead to clinically significant adverse effects that complicate therapy. Knowledge of these adverse effects is important, especially in the management of high-risk or susceptible individuals. The best approach is to establish causality with the drug in question by focused history-taking, treatment review and identification of risk factors. Management mainly focuses on eliminating risk factors, optimisation of pharmacotherapy and supportive measures. Switching/discontinuation of treatment or psychopharmacological interventions are reserved for severe non-responsive adverse effects. Psychotropic drugs should be used cautiously with regular monitoring.

\section{References}

Abers MS, Shandera WX, Kass JS (2014) Neurological and psychiatric adverse effects of antiretroviral drugs. CNS Drugs, 28: 131-45.

Abramowicz M (2008) Drugs that may cause psychiatric symptoms. Medical Letters on Drugs and Therapeutics, 50: 100-3.

Alomar MJ (2014) Factors affecting the development of adverse drug reactions. Saudi Pharmaceutical Journal, 22: 83-94.

Bechstein WO (2000) Neurotoxicity of calcineurin inhibitors: impact and clinical management. Transplant International, 13: 313-26.

Bersani FS, Coviello M, Imperatori C, et al (2015) Adverse psychiatric effects associated with herbal weight-loss products. BioMed Research International, 2015: 120679.

Borovaya A, Olisova 0, Ruzicka T, et al (2013) Does isotretinoin therapy of acne cure or cause depression? International Journal of Dermatology, 52: $1040-52$.

Celano CM, Freudenreich 0, Fernandez-Robles C, et al (2011) Depressogenic effects of medications: a review. Dialogues in Clinical Neurosciences, 13: 103-25.

Cheslack-Postava K, Keyes KM, Lowe SR, et al (2015) Oral contraceptive use and psychiatric disorders in a nationally representative sample of women. Archives of Women's Mental Health, 18: 103-11.

Desai AK (2004) Psychotropic side effects of commonly prescribed medications in the elderly. Primary Psychiatry, 11: 27-34.

Edwards IR, Aronson JK (2000) Adverse drug reactions: definitions, diagnosis, and management. Lancet, 356: 1255-9.

Ernst E (2003) Serious psychiatric and neurological adverse effects of herbal medicines - a systematic review. Acta Psychiatrica Scandinavica, 108: 83-91.

Ernst E (2007) Herbal remedies for depression and anxiety. Advances in Psychiatric Treatment, 13: 312-6.

Ferrando SJ, Levenson JL, Owen JA (2010) Clinical Manual of Psychopharmacology in the Medically III. American Psychiatric Publishing

Gordon JL, Girdler SS (2014) Hormone replacement therapy in the treatment of perimenopausal depression. Current Psychiatry Reports, 6: 517.

Heinrich TW, Marcangelo M (2009) Psychiatric issues in solid organ transplantation. Harvard Review of Psychiatry, 17: 398-406.

Holland JC, Andersen B, Breitbart WS, et al (2013) Distress management: clinical practice guidelines in oncology. Journal of National Comprehensive Cancer Network, 11: 190-209.

Holvey C, Connolly A, Taylor D (2010) Psychiatric side effects of nonpsychiatric drugs. British Journal of Hospital Medicine, 71: 432-6.

Hubbard JR, Levenson JL, Patrick GA (1991) Psychiatric side effects associated with the ten most commonly dispensed prescription drugs: a review. Journal of Family Practice, 33: 177-86.
Huffman JC, Stern TA (2007) Neuropsychiatric consequences of cardiovascular medications. Dialogues in Clinical Neurosciences, 9: 29-45.

Janelsins MC, Kohli S, Mohile SG, et al (2011) An update on cancer- and chemotherapy-related cognitive dysfunction: current status. Seminars in Oncology, 38: 431-8.

Judd LL, Schettler PJ, Brown ES, et al (2014) Adverse consequences of glucocorticoid medication: psychological, cognitive, and behavioral effects. American Journal of Psychiatry, 171: 1045-51.

Kenna H, Poon AW, De Los Angeles CP, et al (2011) Psychiatric complications of treatment with corticosteroids: review with case report. Psychiatry and Clinical Neurosciences, 65: 549-60.

Kersey RD, Elliot DL, Goldberg L, et al (2012) National athletic trainers' association position statement: anabolic-androgenic steroids. Journal of Athletic Training, 47: 567-88.

Keyes KM, Cheslack-Postava K, Westhoff C, et al (2013) Association of hormonal contraceptive use with reduced levels of depressive symptoms: a national study of sexually active women in the United States. American Journal of Epidemiology, 178: 1378-88.

Mehta RD, Roth AJ (2015) Psychiatric considerations in the oncology setting. CA: A Cancer Journal for Clinicians, 65: 299-314.

Meyer RE, Salzman C, Youngstrom EA, et al (2010) Suicidality and risk of suicide - definition, drug safety concerns, and a necessary target for drug development: a consensus statement. Journal of Clinical Psychiatry, 71: e1-21.

Myint AM, Schwarz MJ, Steinbusch HW, et al (2009) Neuropsychiatric disorders related to interferon and interleukins treatment. Metabolic Brain Disease, 24: 55-68

Onder G, Pellicciotti F, Gambassi G, et al (2004) NSAID-related psychiatric adverse events: who is at risk? Drugs, 64: 2619-27.

Pachi A, Bratis D, Moussas G, et al (2013) Psychiatric morbidity and other factors affecting treatment adherence in pulmonary tuberculosis patients. Tuberculosis Research and Treatment, 2013: 1-37.

Parker C (2012) Psychiatric effects of drugs for other disorders. Medicine, 40: 691-5.

Perucca P, Gilliam F (2012) Adverse effects of antiepileptic drugs. Lancet Neurology, 11: 792-802

Raison CL, Demetrashvili M, Capuron L, et al (2005) Neuropsychiatric adverse effects of interferon-alpha: recognition and management. CNS Drugs, 19: 105-23.

Raja M (2012) Impulsive and compulsive behaviors during dopamine replacement treatment in Parkinson's disease and other disorders. Current Drug Safety, 7: 63-75.

Rudorfer MV, Hillefors M (2012) Assessing psychiatric adverse effects during clinical drug development. Pharmaceutical Medicine, 26: 363-94.

Sidhu KS, Balon R (2008) Watch for nonpsychotropics causing psychiatric side effects. Current Psychiatry, 7(4): 61-4, 68-74.

Simons FE, Simons KJ (2008) H1 antihistamines: current status and future directions. World Allergy Organization Journal, 1: 145-55.

Suvada J (2013) Neuropathic and neurocognitive complications of antiretroviral therapy among HIV-infected patients. Neuro Endocrinology Letters, 34 (suppl 1): 5-11.

Tango RC (2003) Psychiatric side effects of medications prescribed in internal medicine. Dialogues in Clinical Neurosciences, 5: 155-65.

Turjanski N, Lloyd G (2005) Psychiatric side-effects of medications: recent developments. Advances in Psychiatric Treatment, 11: 58-70.

US Food and Drug Administration (2008) Information for Healthcare Professionals: Suicidal Behavior and Ideation and Antiepileptic Drugs (1/31/2008: Updated: 12/16/2008). FDA (http://www.fda.gov/Drugs/ DrugSafety/PostmarketDrugSafetyInformationforPatientsandProviders/ ucm100192.htm). Accessed 21 July 2016.

US Food and Drug Administration (2009) Safety Alerts for Human Medical Products: Antiepileptic Drugs. FDA (http://www.fda.gov/Safety/ MedWatch/SafetyInformation/SafetyAlertsforHumanMedicalProducts/ ucm074939.htm). Accessed 21 July 2016
MCO answers

$1 d \quad 2$ c 3 b $\quad 4$ e 5 b 
Warrington TP, Bostwick JM (2006) Psychiatric adverse effects of corticosteroids. Mayo Clinic Proceedings, 81: 1361-7.

Wefel JS, Kesler SR, Noll KR, et al (2015) Clinical characteristics, pathophysiology, and management of non-central nervous system cancerrelated cognitive impairment in adults. CA: Cancer Journal for Clinicians 65: $123-38$.
World Health Organization (2005) The Use of the WHO-UMC System for Standardised Case Causality Assessment. Uppsala Monitoring Centre (http://who-umc.org/Graphics/24734.pdf).

Zahodne LB, Fernandez HH (2008) Pathophysiology and treatment of psychosis in Parkinson's disease: a review. Drugs \& Aging, 25: 665-82.

\section{MCOs}

Select the single best option for each question stem

1 As regards adverse psychiatric effects of corticosteroids:

a severe psychiatric complications are seen in $20-30 \%$ of the patients receiving corticosteroids

b chronic exposure is more commonly associated with hypomanic and manic symptoms than with depressive symptoms

c corticosteroid use is not associated with risk of developing dependence

$\mathrm{d}$ the risk of adverse psychiatric effects increases with the increase in prescribed doses

e psychiatric symptoms occur only in patients with history of psychiatric illness.

2 As regards medications used for treatment of Parkinson's disease:

a the risk of developing adverse psychiatric effects is highest with levodopa and least with anticholinergic drugs

b patients at an early stage of Parkinsonism are more prone to developing adverse psychiatric effects c dopaminergic agents are commonly associated with various impulse control disorders

$\mathrm{d}$ psychosis is a rare adverse effect of dopaminergic agents

e olanzapine is the drug of choice for drug-induced psychosis in patients with Parkinsonism.

3 Depression is a common adverse effect of: a oral contraceptives

b interferon-alpha

c beta-blockers

d ritonavir

e atorvastatin.

4 As regards the assessment of medicationinduced psychiatric adverse effects:

a rechallenge with the suspected problem drug is the most common method of establishing causality

b drugs with narrow therapeutic index have a lower risk of causing adverse psychiatric effects c psychosocial factors do not contribute to the risk of developing adverse psychiatric effects

$\mathrm{d}$ the WHO-UMC system is a tool for the assessment of psychiatric adverse effects in clinical settings

e psychiatric manifestation of the underlying medical disorder is an important differential diagnosis.

5 As regards the management of medication-induced psychiatric symptoms:

a pharmacotherapy with psychotropic agents is the mainstay of treatment

b increasing sensory stimulation and reducing stress in intensive care units may help in reducing the psychiatric symptoms

c the choice of psychotropic agent depends only on its drug-drug interactions with the problem drug

$\mathrm{d}$ discontinuation of the drug or switching to another drug is indicated in most cases

e non-pharmacological approaches have no role in management. 